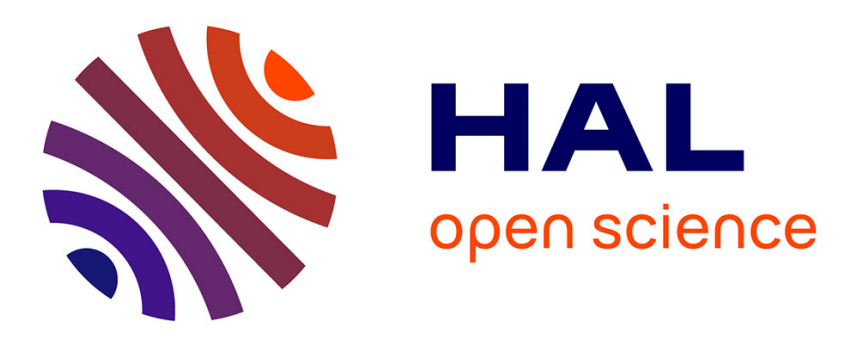

\title{
Scalable Morphological Accessibility of Complex Microstructures
}

Johan Chaniot, Maxime Moreaud, Loïc Sorbier, Jean-Marie Becker, Thierry Fournel

\section{- To cite this version:}

Johan Chaniot, Maxime Moreaud, Loïc Sorbier, Jean-Marie Becker, Thierry Fournel. Scalable Morphological Accessibility of Complex Microstructures. Computational Materials Science, 2022, 203, pp.111062. 10.1016/j.commatsci.2021.111062 . hal-03543639

\section{HAL Id: hal-03543639 \\ https: / hal-ifp.archives-ouvertes.fr/hal-03543639}

Submitted on 26 Jan 2022

HAL is a multi-disciplinary open access archive for the deposit and dissemination of scientific research documents, whether they are published or not. The documents may come from teaching and research institutions in France or abroad, or from public or private research centers.
L'archive ouverte pluridisciplinaire HAL, est destinée au dépôt et à la diffusion de documents scientifiques de niveau recherche, publiés ou non, émanant des établissements d'enseignement et de recherche français ou étrangers, des laboratoires publics ou privés. 


\title{
Scalable morphological accessibility of complex microstructures
}

\author{
Johan Chaniot $^{\mathrm{a}, \mathrm{b}, *}$, Maxime Moreaud ${ }^{\mathrm{a}, \mathrm{c}}$, Loïc Sorbier ${ }^{\mathrm{a}}$, Jean-Marie Becker ${ }^{\mathrm{b}}$, Thierry Fournel ${ }^{\mathrm{b}}$ \\ ${ }^{a}$ IFP Energies nouvelles, Rond-point de l'échangeur de Solaize, BP 3, 69360 Solaize, France \\ ${ }^{b}$ Univ. Lyon, UJM-Saint-Etienne, CNRS, Institute of Optics Graduate School, Laboratoire Hubert Curien UMR5516, \\ F-42023 St-Etienne, France
}

${ }^{c}$ MINES ParisTech, PSL-Research University, CMM, 35 rue Saint Honoré, 77305 Fontainebleau, France

\begin{abstract}
This paper addresses the descriptors-based characterization of dense 3D microstructures using the unifying concept of accessibility, mixing local shape features with global topology. Underlying percolation and constrictivity features are jointly considered by probing the connected components of the microstructure with structuring elements with increasing sizes. Adapted morphological operations are combined to provide a scalable protocol embedding suitable descriptors applied on accessible volumes, yielding a sharp discrimination power. The suggested framework named A-protocol can efficiently analyze complex microstructures by applying a stratified sampling for the selection of paths' endpoints, when connected. It stops when percolation ends, at a critical radius value. The A-protocol is tested on Cox multi-scale Boolean models using the Euler number as an arbitrarily chosen embedded descriptor. This computational protocol is available in the open access software environment plug im!
\end{abstract}

Keywords: Feature-based vector; Accessibility; Percolation; Constrictivity; Mathematical Morphology; Porous Network

\section{Symbols}

The main symbols used in this article are exposed and defined in the following list.

- $X$ : the microstructure of interest,

- $\epsilon_{r}(X)$ : the eroded set of $X$ using a sphere $B(r)$ of radius $r$ as structuring element,

- $D_{\max }$ : maximal distance value of the distance map used for computing the erosion,

- $r_{c}$ : the critical radius of $X$,

- $\rho_{c}$ : the critical percolation threshold of the complementary set of $\epsilon_{r_{c}}(X)$,

- $\beta$ : the constriction factor,

- $V_{v_{A}}$ : the accessible volume fraction of $\epsilon_{r}(X)$, 
- $N_{C}$ : the number of cavities,

- $V_{C}$ : the average volume of cavities,

- $S$ : the set of random points $p_{i}$ in $X$,

- $t$ : the threshold applied to the number of connected paths $n_{\text {paths }}$ between points $p_{i}$,

- $\chi$ : the Euler-Poincaré characteristic or Euler number.

\section{Introduction}

The analysis of interconnected networks is of paramount interest for many applications $[1,2,3]$, especially in materials science $[4,5,6]$. The structural characterization of materials enables valuable connections with physicochemical properties, especially in the analysis of porous media $[7,8]$. Due to the complexity of some interconnected networks, named microstructures when real media are considered, their structural characterization often requires combination of various operators, named descriptors, focusing either on local -morphological- or global -topological- features $[9,10,11]$.

Among topological measures, connectivity notion is considered in various descriptors, as connectivity number and Euler number, and concepts too, as percolation and accessibility [1, 12,3]. This latter turned out to have a central interest on various experiments considering hindrance phenomenon, making use of different flowing particles and measurement means $[13,14,15,16]$. Indeed, hindrance in porous media is crucial as the size of flowing particles can impact transport properties, especially when it has the same order of magnitude as the pores dimensions [17, 18]. In the digital domain, after 3D microstructure segmentation, hindrance could be addressed in the scope of morphological accessibility, defined here by combining connectivity seen through percolation, i.e., ability to connect, with constrictivity, i.e., strength of bottleneck effect [19]. Consequently, this morphological vision of accessibility is purely structural, no physicochemical phenomenon is considered.

On the one hand, percolation as a theory considers accessibility in a specific way [20,21], indicating the existence of a connected path totally included in the pattern, connecting a given entry to a given exit (Fig.1(A-D)). This binary vision of connectivity, linked to global topological notions, received several numerical implementations of distinct types, which are not constrained to a single binary value [22]. On the other hand, constrictivity, introduced by Petersen [23], highlights local hindrance through bottleneck effects quantification, usually represented by a scalar value named constriction factor $\beta$ [24]. Originally defined for a single path or a synthetic pore (Fig.1(e)), both concepts have been extended to whole microstructures, mainly using statistical representations $[25,26]$.

Similar ideas in literature address accessibility, reachability or penetrability by taking into account the local shape together with global assessments of proper descriptors, considering implicitly or explicitly percolation and constrictivity concepts. These works are of different types, involving either experimental analysis or numerical simulations of transports of any kind or purely digital morphological characterization, or a mix of the latter [27, 28, 17, 29, 30, 18, 19].

Wernert et al. [17] tackle this issue with experimental analyses only, focusing on porosity, diffusion and tortuosity as a function of molecular size to predict transport properties of porous materials. Skaug et al. [18] make use of nanoparticles tracking to study diffusion, highlighting the dependency of accessibility on particle size too. The needed improvement of characterization 


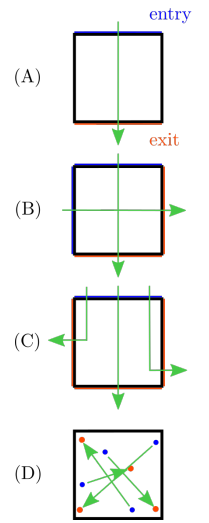

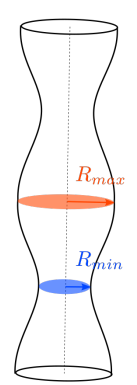

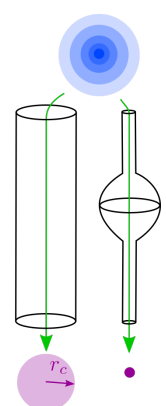

(f)

Figure 1: (A-D) Four forms of percolation: (A) directional percolation (between two opposite faces), (B) multidirectional percolation (in at least one direction), (C) adirectional percolation (between distinct faces) [33], and (D) stochastic point percolation [34] (between random points). Illustration of (e) constrictivity and (f) accessibility with critical radius $r_{c}$ (see Section 2).

process is justified in [29], by the advances in computation and visualization technologies and not in analysis methods. As they focus on multi-scale transport models, they propose to consider various descriptors according to the pore scale using morphological erosions [31, 32]. Vogel [27] already mentions the issue of the geometric complexity of real microstructures. He uses the Euler-Poincaré characteristic, or Euler number, to quantify the pore connectivity which is considered as a function of the pore diameter, defining a connectivity function of the pore space. As in his article in 2002 [28], morphological openings, erosion followed by dilation, with different sizes of structural elements are used for pore size distribution assessment. For their part, Ohser et al. [30] explicitly take into account percolation, introducing a percolation probability depending on pores' width to describe penetrability. Similarly to [19], only erosions are considered, but to assess the porosity percolation probability instead of the reachable volume fraction, as it is done in [19].

This diversity of disciplines attests to the interest of the whole materials science community about this concept, highlighting the need of improving the set of solutions for materials analysis. Moreover, although these studies are of distinct type, each one assesses the impact of accessibility over different descriptors of porous media; spherical probes of different sizes travelling through the network could be a common basis for accessibility, as illustrated in Fig.1(f). Nevertheless, to our best knowledge, in literature, no numerical solution such as computational protocol, is available in order to take into account accessibility, evaluating its impact on any proper descriptor to the application considered.

The main contribution of this article is a novel scalable framework considering morphological accessibility, being a solution to the issue mentioned above. Thus named, the A-protocol is suitable for any specific application-dependent descriptors, extending it in an easily interpretable manner, as shown below with the Euler number as an example of possible embedded descriptor, while assessing the effect of pore's/probe's size on it. Moreover, as complex microstructures are targeted, usual computational methods to assess percolation have to be improved; an example is presented in Fig.7, being complex because of both, the porous network geometry and the random 
global shape of the observed sample. Finally, the A-protocol, being freely available in plug im! [35], provides novel solutions, targeting a more exhaustive characterization of microstructures. Throughout the article, the term "accessibility" will mean "morphological accessibility".

The computational protocol is proposed in Section 2, providing a versatile methodology addressing accessibility via 3D patterns. Its effectiveness is tested on Cox multi-scale Boolean models, focusing on the stochastic form of point percolation and point accessibility: the results quantifying local anisotropy and global heterogeneity are reported in Section 3. The conclusion is drawn in Section 4.

\section{Accessibility to complex microstructures}

First, discussions on percolation and on constrictivity define our vision of these two concepts, which are jointly considered in our definition of a ccessibility. Second, the A-protocol is defined and illustrated. Finally, the relationships connecting the critical radius to constrictivity and to percolation are given.

\subsection{From percolation to constrictivity}

Percolation is usually assessed utilizing a connected components labeling, assigning a specific label to each connected component [36]. In order to remove the constraint of choosing planar sections as entries and exits, Chaniot et al. [34, 37] make use of a stratified sampling [38], yielding to a stochastic consideration of percolation, assessed between random points or vertices, in a certain way akin to graph theory $[5,11]$. Therefore, the A-protocol defined below, is able to support the different forms of percolation illustrated in Fig.1(A-D), including the stochastic version of percolation (Fig.1(D)) dedicated to microstructures as complex as the one in Fig.7. This form of percolation is named stochastic point percolation.

As mentioned in the introduction, the accessibility is defined by considering j ointly percolation and constrictivity; this last one is illustrated in Fig.1(e) and can be quantified by the ratio $R_{\min }^{2} / R_{\max }^{2}$ [26]. Morphological openings or erosions, considering spheres as structuring elements with increasing sizes, can be utilized for characterization purposes according to pores' or particles' size. Erosion is adapted to topological measurements, while opening is suitable for geometric ones. For computation time purposes, the erosion is considered in this article, efficiently computed using distance transform [39], quantifying in a certain way constrictivity by disconnections consideration.

\subsection{The A-protocol}

The definition of a protocol devoted to accessibility is motivated by the transfer of the concept into a scalable digital framework embedding relevant descriptors, with efficiency concerns, which are then extended in order to be applied on complex microstructures.

The A-protocol is an iterative method taking into account all bottlenecks of a microstructure by considering increasing radii $r$ for spheres $B(r)$, i.e., the structuring elements of the erosions. At each iteration, percolation is jointly verified a fter labeling connected c omponents, and predefined embedded descriptors are a ssessed. Some cavities defined as the resulting non-accessible connected components for a probe of a given size, are formed due to the hindrance caused by the probe dilation. Geometrically, the larger the radius, the more there will be vanished volumes. Topologically, the trajectories between any two remaining points of the microstructure, lengthen until they are closed. The A-protocol ends when the critical radius $r_{c}$ is reached, i.e., the radius 
of the biggest percolating spherical probe $B\left(r_{c}\right)$.

As the A-protocol is a cumulative step by step process, if residues descriptors are considered, as cavities, the value of the descriptor for a specific size is equal to the sum over these residues from $r=0$ to the given size.

Sampling 3D patterns is optional, but required in the context of stochastic point percolation giving rise to stochastic point accessibility, that we define herebelow,

Definition 1. Stochastic point accessibility: Let $\epsilon_{r}(X)$ be the eroded set of microstructure $X$ using a sphere $B(r)$ as structuring element. Let $S=\left\{p_{i}\right\}$ be a set of $N$ random points such that $S \subset \epsilon_{r}(X)$. A connected component of $\epsilon_{r}(X)$ is said to percolate if at least a number $t$ of connected paths exists between some pairs of points $\left(p_{i}, p_{j}\right), i \neq j$. Eroded set $\epsilon_{r}(X)$ percolates if there exists at least a percolating connected component.

Let $C c_{r}$ be the union of the percolating connected components of $\epsilon_{r}(X)$. If $C c_{r} \neq \emptyset, X$ is said to be accessible for a probe $B(r)$.

In order to comply with usual definitions, we take $t=1$; for a connected component to be considered as accessible, it suffices that a connected path has been found. The stochastic point accessibility allows the A-protocol to be applied on any microstructures, reflecting the flowing particles in the physical domain.

The focus being mainly on topological measurements and computation time efficiency, the erosion operation is chosen instead of opening, as mentioned above, and in the case of stochastic point accessibility, $S$ is defined once for all at the initialization step.

The A-protocol illustrated in Fig.2 on a synthetic structure, is formally given by the algorithm below. The estimates of accessible volume fraction $\hat{V}_{v_{A}}$, number of cavities $\hat{N}_{C}$, and average volume of cavities $\hat{V}_{C}$ are simultaneously assessed until the critical radius $\hat{r}_{c}$ is reached. These estimates are inherent to the A-protocol definition and have to be distinguished from the arbitrary embedded descriptors which are selected by the user. Let us note that the skeleton of microstructure $X$ [40] could be considered, but would lead to increase the bias over morphological descriptors as volume fraction assessments.

\subsection{Constrictivity, percolation and critical radius}

Constriction factors $\beta$ quantifying bottlenecks, are scalar values obtained from comparison of minimal and maximal cross sections [24, 41]. A specific definition consists in computing the squared ratio of the minimal to the maximal radius, which could be translated in our case by using the critical radius $r_{c}$ and the maximal radius, i.e. the maximal distance value $D_{\max }$ obtained from the distance map used for the iterative erosions.

$$
\beta=\left(\frac{r_{c}}{D_{\max }}\right)^{2}
$$

To avoid border effects, leading to overestimation of the maximal radius, a maximal distance $D_{\text {max }}$ is computed over the whole image. A second computation is performed with the condition that the location of the maximal distance $D_{\max }$ has to be further from the border than $D_{\max }$. Moreover, all the computations in the Results section are performed on points further from the border than $D_{\max }-D_{\max }$.

The original definition of $\beta$ being for a unique pore, this definition is a novel alternative to 
Input: Pattern $X$; embedded descriptor $\left\{D_{k}\right\}$; (optional) sampling points set $S$

Output: Estimates $\hat{V}_{v_{A}}, \hat{N}_{C}, \hat{V}_{C}$ and embedded descriptor $\left\{\hat{D}_{k}\right\}$, as functions of $r$; critical radius $\hat{r}_{c}$

Initialization: $r \leftarrow 0$

while Percolation do

A-1. Connected components labeling $X \leftarrow\left\{c c_{i}\right\}$ (Fig.2(1))

A-2. Percolation assessment and computation of $C c_{r}$ (Fig.2(2))

if Percolation then

A-3. Computation of estimates $\hat{V}_{v_{A}}(r), \hat{N}_{C}(r), \hat{V}_{C}(r)$ and embedded descriptor $D_{k}(r)$ over $C c_{r}$

A-4. Erosion, $X \leftarrow \epsilon_{1}(X)$ (Fig.2(4))

Incrementation: $r \leftarrow r+1$

end

end

Critical radius: $\hat{r}_{c} \leftarrow r-1$

Algorithm 1: A-protocol

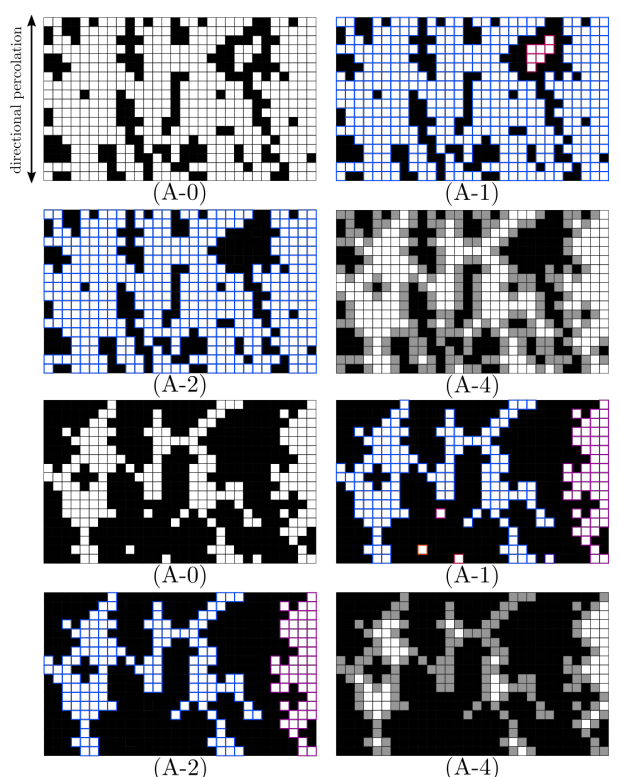

Figure 2: Analysis of a synthetic structure (in white) by the A-protocol; directional percolation and $\hat{r}_{c}=1$. (A- 0 ) shows the pattern state at the starting of an iteration, (A-1) connected components labeling, each label being associated with a color, (A-2) percolation assessment with deletion of non percolating connected components, (A-3) (not displayed) computation of descriptors, (A-4) morphological erosion using $B(1)$, deleted pixels being in gray. 
extend it to a whole network.

By definition, the critical radius $r_{c}$ is linked to the critical percolation threshold $\rho_{c}$, which can be assessed using a statistical approach [25] when stochastic models are considered, then defined as the volume fraction where exactly $50 \%$ of the realizations percolate.

Boolean models [42, 43, 44], a specific stochastic model, are based on a Poisson Point process of intensity $\theta$ [45]. Random primary grains $A^{\prime}$ (overlapping allowed) are located at Poisson points $x_{k}$. $A^{\prime}$ and $\theta$ define the Boolean model $A$, being equal to the union of grains $A^{\prime}$. Eq. 2 shows dependency of $\theta$ with respect to the average volume $\bar{V}\left(A^{\prime}\right)$ of the grains and to the total volume fraction $V_{v}$.

$$
1-V_{v}=\exp \left(-\theta \cdot \bar{V}\left(A^{\prime}\right)\right)
$$

Let $A_{r}=A \oplus B(r)$ be a Boolean model defined as the dilation of $A$ by $B(r)$, and $V_{v, r}$ be the volume fraction of $A_{r}$. As we focus on the complementary set of $A$, this operation represents the erosion of the microstructure.

As $V_{v, r} \geq V_{v}$, there exists a function $\alpha, \forall r \geq 1 \alpha(r) \geq 1$, such that $\bar{V}\left(A_{r}^{\prime}\right)=\alpha(r) . \bar{V}\left(A^{\prime}\right)$. Therefore, with eq. $2,1-V_{v, r}=\left(1-V_{v}\right)^{\alpha(r)}$.

Henceforth, $r_{c}$ is a limit value of $r$, obtained when $1-V_{v, r}$ is equal to the threshold $\rho_{c}$ of the eroded complementary set. Therefore, there exists $\alpha_{c}=\alpha\left(r_{c}\right)$ and,

$$
\begin{aligned}
& \rho_{c}=1-V_{v, r_{c}} \\
& \rho_{c}=\left(1-V_{v}\right)^{\alpha_{c}} \\
& \alpha_{c}=\ln \left(\rho_{c}\right) / \ln \left(1-V_{v}\right) .
\end{aligned}
$$

In the case of a Boolean model of sphere of radius $R, A^{\prime}=B(R)$, therefore $\alpha_{c}=\bar{V}\left(A_{r_{c}}^{\prime}\right) / \bar{V}\left(A^{\prime}\right)=$ $\left(\left(R+r_{c}\right) / R\right)^{3}$ and,

$$
r_{c}=\left(\sqrt[3]{\ln \left(\rho_{c}\right) / \ln \left(1-V_{v}\right)}-1\right) \cdot R .
$$

The same rationale with the Cox multi-scale Boolean models, as considered below, is proposed. The total volume fraction of primary grains $V_{v, T O T}$ is defined by,

$$
V_{v, T O T}=V_{v, I N C} \cdot V_{v, I N}+\left(1-V_{v, I N C}\right) \cdot V_{v, O U T}
$$

with $V_{v, I N C}$ the volume fraction of inclusions or aggregates, with higher density, $V_{v, I N}$ the volume fraction of grains inside aggregates, and $V_{v, O U T}$ the volume fraction of grains outside aggregates. Eq. 2 is valid for each volume fraction, leading to,

$$
\rho_{c}=\left(1-V_{v}\right)^{\alpha_{c}}-\left(1-V_{v, I N C}\right) \cdot\left(\left(1-V_{v}\right)^{\alpha_{c}}-\left(1-V_{v, \text { OUT }}\right)^{\alpha_{c}}\right) .
$$

These equations define our critical radius in the specific cases of Boolean models and Cox multi-scale Boolean models, while highlighting the relationship between the critical percolation threshold $\rho_{c}$ and the critical radius $r_{c}$.

\section{Results}

For testing the A-protocol and illustrating its properties on complex microstructures, stochastic models or numerical twins are considered. In particular, we insist on the stochastic point accessibility. For these purposes, Cox multi-scale Boolean models which are suitable for statistical 
analyses of computational methods and usual investigation of stereological operators are used [46]. Therefore, the A-protocol's behavior analysis with respect to specific features is practicable; impact of the grains' anisotropy and the geometric heterogeneity over the accessibility to the microstructure. This is preceded by the presentation of the stochastic models considered, and by the comparative study of the parametric stochastic point percolation with the other forms of percolation.

\subsection{Stochastic models}

Boolean models are first considered in order to generate homogeneous microstructures made of isotropic or anisotropic grains $A^{\prime}$, located at Poisson points. A homogeneous microstructure is defined as a structure possessing a unique scale of grains' density, quantified by a unique volume fraction $V_{v}$. Consequently, hereafter, they are defined by this single volume fraction of grains $V_{v}$. Multi-scale microstructures can be modeled by using Cox multi-scale Boolean models [47, 48], simulating heterogeneous materials with aggregates or inclusions, i.e., areas of higher density of grains. In the following, two-scale models are considered, defined by three volume fractions: $V_{v, I N C}, V_{v, I N}$ and $V_{v, O U T}$.

Boolean models of spheres (Fig.3(a)) and of spherocylinders with random orientations (Fig.3(b)) are generated; $V_{v}$ is fixed at 0.4 for both models, having a similar fixed average volume of grain, $\bar{V}\left(A^{\prime}\right)=4188.8$ for spheres and $\bar{V}\left(A^{\prime}\right)=4215.0$ for spherocylinders.

1. sphere $R=10$,

2. spherocylinder $R=5$ and $L=47$.

Cox multi-scale Boolean models of platelets are considered; platelets' shape is fixed (Fig.3(c)): $L=6, l=5$ and $H=3$, aggregates' size and volume fraction are fixed too: $R_{I N C}=10$, $V_{v, I N C}=0.5$, as well as the total volume fraction of grains $V_{v, T O T}$ which is equal to 0.4.

3. $V_{v, I N}=0.5$ and $V_{v, O U T}=0.3$,

4. $V_{v, I N}=0.6$ and $V_{v, O U T}=0.2$,

5. $V_{v, I N}=0.7$ and $V_{v, O U T}=0.1$.

40 realizations of size $400^{3}$ of each model are generated (Fig.3). The A-protocol is applied to the complementary set of the grains union (black areas in Fig.3), representing the microstructure of interest. Consequently, a volume fraction of grains $V_{v}$ equal to 0.4 leads to an accessible volume fraction $V_{v_{A}}$ equal to 0.6 , if and only if the whole microstructure is accessible.

Confidence intervals with $95 \%$ confidence level, represented by vertical bars in the results dis-

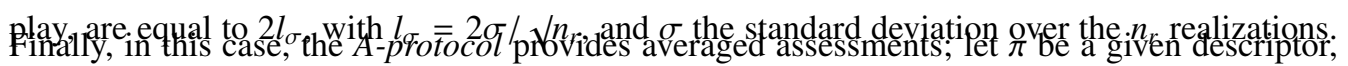
then $\hat{\pi}$ is an averaged estimator over all realizations.

\subsection{Stochastic point accessibility}

Let us now restrict our attention to the model (1). Only $\hat{r}_{c}$ and $\hat{V}_{v_{A}}$ are displayed in Tab. 1 and Fig.4. As a global statement, the low values of $l_{\sigma}$ attest to the representativity of the considered volume, i.e. the realizations' volume times the realizations number. Confidence intervals of all curves are represented, but too small to be visible before a certain value; the larger the radius $r$, the bigger the representative volume element, the lower the representativity [49].

The stratified s ampling, as defined in [34], is a parametric me thod, wi th $N_{T}$ and $N$ the target number of random points given by the user and the final number of random points, respectively. 

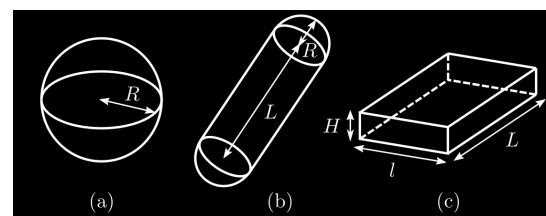

Boolean models

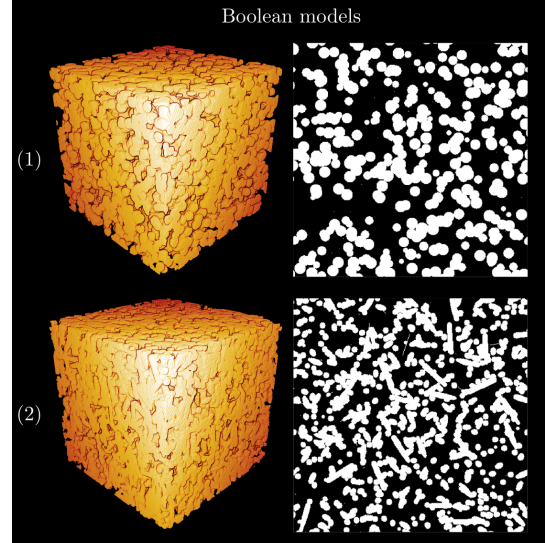

Cox multi-scale Boolean models

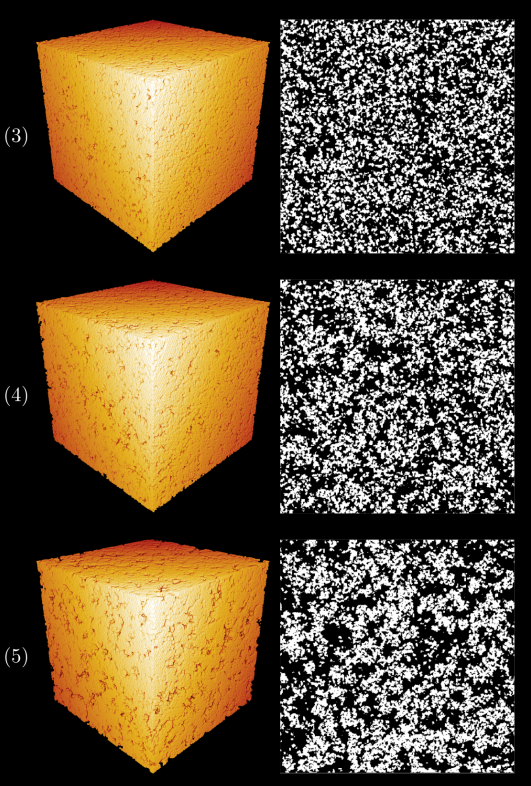

Figure 3: The different grain's shapes used in this paper: (a) sphere, (b) spherocylinder and (c) platelet. Volume representation and 2D slice of a realization of each Boolean model: (1) spheres and (2) spherocylinders, and Cox multi-scale Boolean models of platelets: (3) $V_{v, I N}=0.5$, (4) $V_{v, I N}=0.6$ and (5) $V_{v, I N}=0.7$. Volumes generated and rendered using [35]. 
Table 1: $1^{\text {st }}$ line: $\hat{r}_{c}$ for the stochastic accessibility with different $e$ values. $2^{\text {nd }}$ line: $\hat{r}_{c}$ for the various accessibility types. Display of $l_{\sigma}$, the half length of the confidence interval, with $95 \%$ confidence level.

\begin{tabular}{|c|c|c|c|}
\hline Model & Percolation & $\hat{r}_{c}$ & $l_{\sigma}$ \\
\hline \multirow{5}{*}{ (1) Sphere } & Stochastic $e=133$ & 9.175 & 0.311 \\
& Stochastic $e=100$ & 9.050 & 0.237 \\
& Stochastic $e=80$ & 9.400 & 0.266 \\
& Stochastic $e=66$ & 10.950 & 0.531 \\
\hline \multirow{5}{*}{ (1) Sphere } & $\mathrm{A}$ & 8.900 & 0.096 \\
& $\mathrm{~B}$ & 8.975 & 0.050 \\
& $\mathrm{C}$ & 13.950 & 0.423 \\
& $\mathrm{D}$ & 9.050 & 0.237 \\
\hline
\end{tabular}

None of them is meaningful because they are both dependent of the size of the considered images, but the length of the cubic sub-images' edges $e$ is. During the stratified sampling process, the image is cut into cubic sub-images of exact same volume in which one point is randomly drawn; $e$ is the length of their edge, then equal to the average Euclidean distance between two neighbouring points. The purpose is to assess its optimal value with respect to an arbitrary reference. Hereafter, we choose the percolation (A) as the reference $\left(\hat{r}_{c, r e f}, \hat{V}_{v_{A, r e f}}\right)$.

The A-protocol with stochastic point accessibility, is analyzed as a function of $e=$ $\{133,100,80,66\}$ (Fig.4(a)). One can notice some slight differences with (A) due to negligible volumes (Fig.4 bottom right); Fig.4(a) shows the similarities of $\hat{V}_{v_{A}}$ whatever $e$. The best assessment of $\left(\hat{r}_{c, r e f}, \hat{V}_{v_{A, r e f}}\right)$ is reached for $e=100(N=64)$, as shown in Tab.1. The same process is performed on each model showing a necessary refinement for the more complex ones; (1) $e=100$, (2) $e=100$, and (3) $e=100$, (4) $e=80(N=125)$, (5) $e=80$. Hereafter, these values are considered in percolation (D).

Let us compare now the four percolation forms (Fig.4(b)). Globally, the curves are quite similar whatever the percolation. The considered models being isotropic, the percolations (A) and (B) exhibit very similar results, as for the percolation (D), which is highlighted in Tab.1 too. Nevertheless, the percolation (C) having a less constrained definition (percolation between faces with a common edge is allowed), it overestimates (A) in terms of $\hat{r}_{c}$ (Tab.1) and $\hat{V}_{v_{A}}$, but the low representativity of the remaining connected components is shown with the bigger confidence intervals when $\hat{r}_{c, \text { ref }}$ is passed. The accessible volume in Fig. 4 bottom, places in evidence the similarities and differences; in particular with (C) for $r=10$, where the remaining connected components are placed at edges and corners of the cubic image.

\subsection{Microstructures characterization}

The focus is now on the A-protocol with stochastic point accessibility only. Although $\hat{r}_{c}$ is sufficient to discriminate the models in Tab.2, additional information is provided by the constriction factor $\hat{\beta}$, and also the behavior of the estimates, inherent to the A-protocol, and of the embedded descriptor, being the Euler number $\chi$.

The Euler number $\chi$ is arbitrarily selected as an example of embedded descriptor. $\chi$ is one of 


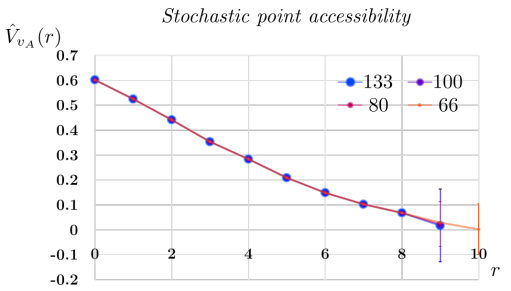

(a)

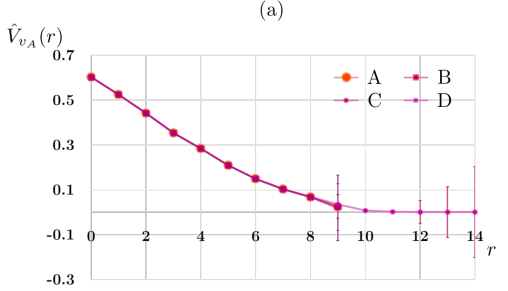

(b)

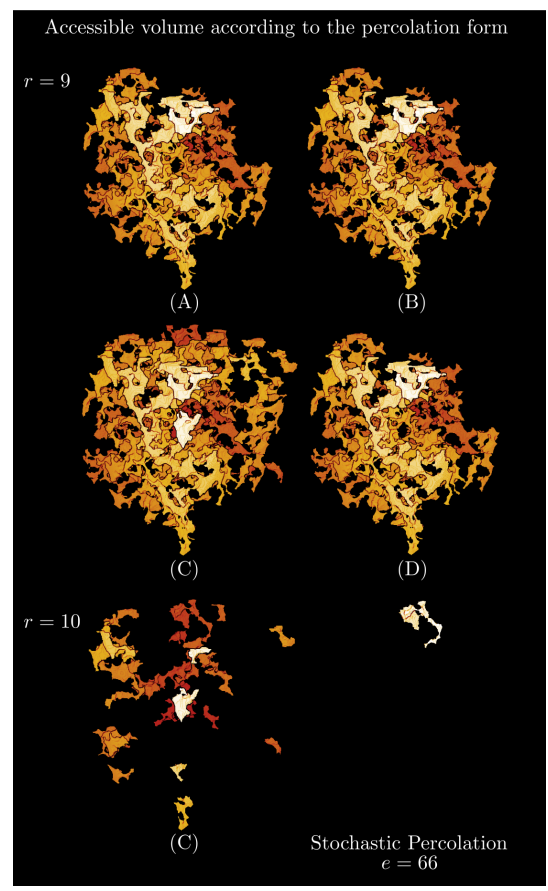

Figure 4: $\hat{V}_{v_{A}}$ for (a) the stochastic percolation $(e \in\{133,100,80,66\})$ and (b) the four forms of percolation ((A), (B), (C), (D)), applied to Boolean models (1). Confidence intervals, with $95 \%$ confidence level, are represented by vertical bars. The accessible volume of a realization of the Boolean model (1) for specific $\mathrm{r}$ adii: $r=9=\hat{r}_{c, \text { ref }}$ and $r=10$. Volumes rendered using [35]. 


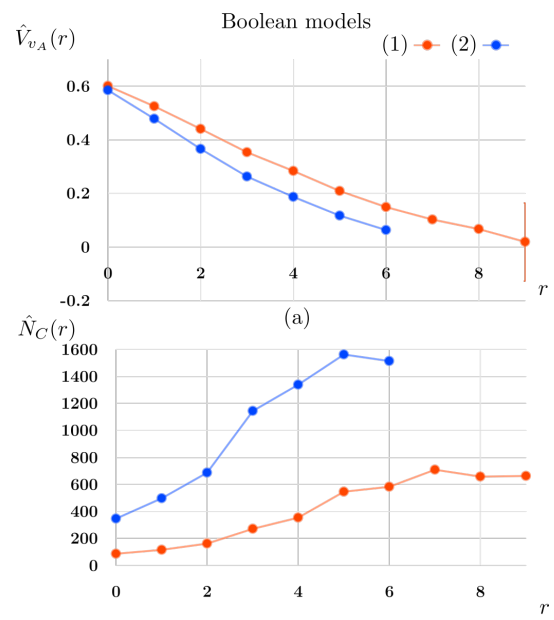

(b)
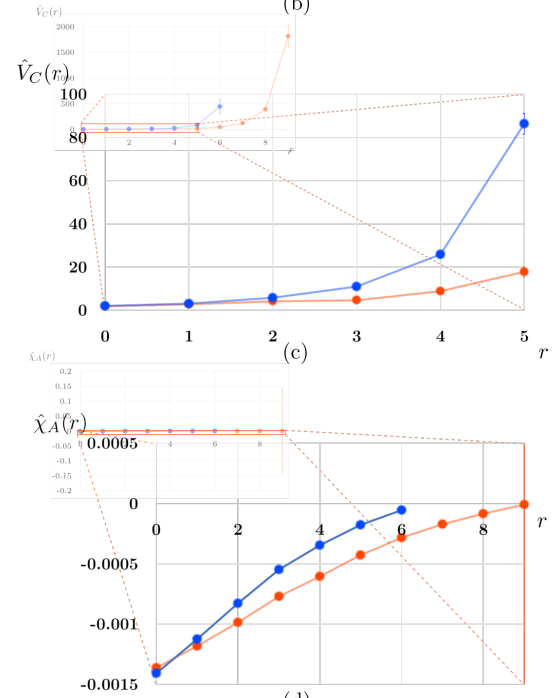

(d)

Figure 5: A-protocol with stochastic point accessibility applied to the Boolean models: (a) $\hat{V}_{v_{A}}$ and (b) $\hat{\chi}_{A}$ ((b) is an enlargment, orange rectangle, of original curves, small and blurred). Confidence intervals, with $95 \%$ confidence level, are represented by vertical bars. 
Table 2: $\hat{r}_{c}$ and $\hat{\beta}$ for the stochastic point accessibility with optimal $e$ values, for all models. Display of $l_{\sigma}$, the half length of the confidence interval, with $95 \%$ confidence level.

\begin{tabular}{|c|c|c|c|c|}
\hline Model & $\hat{r}_{c}$ & $l_{\sigma}$ & $\hat{\beta}$ & $l_{\sigma}$ \\
\hline (1) Sphere & 9.050 & 0.237 & 0.182 & 0.012 \\
\hline (2) Spherocylinder & 6.325 & 0.166 & 0.123 & 0.007 \\
\hline (3) $V_{v, I N}=0.5$ & 2.000 & 0.000 & 0.077 & 0.002 \\
\hline (4) $V_{v, I N}=0.6$ & 2.575 & 0.158 & 0.096 & 0.011 \\
\hline (5) $V_{v, I N}=0.7$ & 3.575 & 0.174 & 0.118 & 0.011 \\
\hline
\end{tabular}

the main topological descriptor of microstructures, characterizing their connectivity or interconnectivity [50, 51, 52]. This feature, connected to the Minkowski functionals [53, 42, 43], has been the subject of various works $[54,55,56,57] . \chi$ can be expressed as a combination of some classic topological measures,

$$
\chi=n_{C C}-c_{R}+h
$$

with $n_{C C}$ the number of isolated objects or connected components, i.e. pores or set of pores, $c_{R}$ the number of redundant connections and $h$ the number of cavities, equal to zero in our case as they are removed at each iteration. Consequently, a negative value represents a high interconnectivity and a positive value represents a low interconnectivity.

Hereafter, $\chi$ is assessed as a function of the probe's radius; its extension produced by the A-protocol, the accessible Euler number, and its numerical estimator are named $\chi_{A}$ and $\hat{\chi}_{A}$, respectively.

First, let focus on the models (1) and (2), i.e., the Boolean models, which are clearly discriminated in Fig.5. Although the anisotropy of the spherocylinder, characterized by its morphological diameter too, provides a smaller value of $\hat{r}_{c}$, its global constriction factor $\hat{\beta}$ is smaller than for the spheres (Tab.2). Moreover, as shown in Fig.5(a-c), this grains' anisotropy strongly induces the creation of cavities, which are bigger too, when $r$ increases; the more pronounced the local bottleneck effects, the faster the pores' closing, the faster the decrease of accessible volume. Fig.5(a) displays this decrease of volume accessibility with the grains' anisotropy. Finally, these phenomena linked to the higher anisotropy of spherocylinders lead to a faster decrease of interconnectivity, i.e., $\chi_{A}$ tends faster toward zero from an initial negative value (Fig.5(d)).

Considering the models (3-5), Fig.6(a-d) underlines the good discrimination between the distinct heterogeneities. Indeed, heterogeneity naturally involving cavities formation (Fig.6(b-c)). For $V_{v, T O T}$ fixed and $V_{v, I N C}=0.5$, g lobally, the $m$ ore heterogeneous the $m$ icrostructure, the less dense the exterior of aggregates, the slower the porous network closes because of the enlarging of the pores size, decreasing the strength of local bottleneck effects outside aggregates. Nevertheless, the more heterogeneous the microstructure, the more dense the aggregates, the faster the aggregates close, increasing the strength of local bottleneck effects inside aggregates. Consequently, with these two opposite phenomena, the global bottleneck effect increases with heterogeneity; the more heterogeneous the microstructure, the bigger $\hat{\beta}$. This second case attests also to the slower decrease of interconnectivity with heterogeneity for large enough particles, if 
the outside of the aggregates percolates (Fig.6(d)).

At a finer scale, the A-protocol puts in evidence the classification reversal of topological measures $\hat{N}_{C}, \hat{V}_{C}$ and $\hat{\chi}_{A}$, attesting to the heterogeneity of these models (Fig.6(b-d)). Indeed, this behavior, corresponding to the very "moment" of the aggregates closing, provides models' discrimination; in particular between homogeneous (models (1-2)) and heterogeneous materials (models (3-5)), highlighted by the comparison of Fig.5 and Fig.6. Consequently, together, these cases bring novel information about heterogeneity detection, while giving intels about the impact of grains' anisotropy over isotropic and homogeneous models. Furthermore, they highlight the improvement in sensitivity of the embedded descriptors, which are extended by accessibility consideration.

\section{Conclusion}

The A-protocol combines state-of-the-art definitions dynamically considering the accessibility, aiming to extend any given numerical descriptor definition with topological notions. The $A$ protocol integrates all bottleneck effects, detected by the spherical probes with increasing radii, and gives rise at the end to estimates as the critical radius, the accessible volume fraction and the number and the average size of cavities. The A-protocol is illustrated using Cox multi-scale Boolean models, validating its interest as a new "extractor" of morphological and topological information. The A-protocol with the stochastic point accessibility, providing a less restrictive method through the existence of a connected path between random points, is discussed. This analysis attests to the similarities of this definition with the most common definition in the literature, yielding a good estimator. The Euler number is then considered, illustrating together with the estimates, the enhancement of discriminative power when characterizing multi-scale microstructures; anisotropy discrimination and heterogeneity detection and discrimination.

The A-protocol exacerbating initially imperceptible differences, is able to characterize microstructures whatever their complexity, inherent to the materials and/or stemming from the image acquisition device, thanks to the stochastic point accessibility. Consequently, real materials as the $\gamma$-alumina (Fig.7), can be characterized with any proper morphological descriptors, extending the work of Moreaud et al. [58] and opening wide application perspectives. Also, a deterministic extension of the A-protocol will be defined, similarly to [59], yielding to a deterministic point accessibility. Finally, a grayscale extension would allow the consideration of continuous fields, as mentioned in [28].

The A-protocol procedure is available in the open access software environment plug im! [35].

\section{Data Availability}

The data, i.e. binary images, required to reproduce these findings can be generated using the open access software environment plug im! at www.plugim.fr; all the needed parameters are explicitly given in the article. Moreover, the numerical tools used for any image processing considered are also freely available in this same website.

\section{Acknowledgments}

We would like to thank the reviewers for their relevant and constructive comments. 


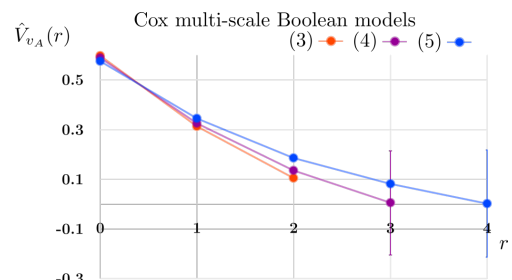

$-0.3+(a)$
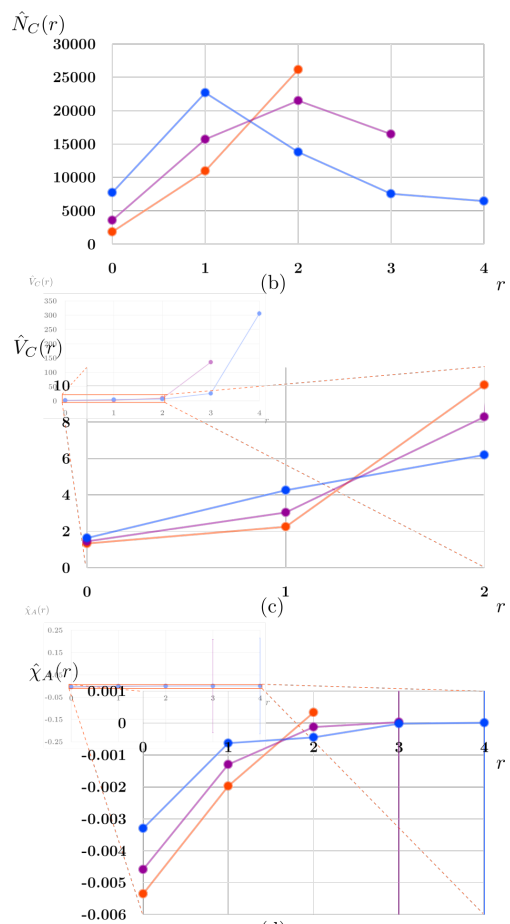

(d)

Figure 6: A-protocol with stochastic point accessibility applied to the Cox multi-scale Boolean models: (a-c) A-protocol estimates and (d) $\hat{\chi}_{A}$ ((c-d) are enlargments, orange rectangle, of original curves, small and blurred). Confidence intervals, with $95 \%$ confidence level, are represented by vertical bars.

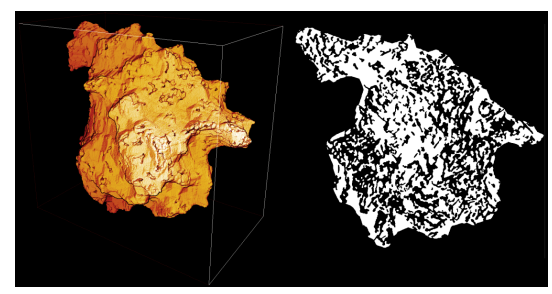

Figure 7: A sample of a specific $\gamma$-alumina, obtained by electron tomography, reconstructed, filtered, and segmented: (left) the porous volume, (right) a slice. Volumes rendered using [35]. 


\section{References}

[1] A. Kruglova, M. Engstler, G. Gaiselmann, O. Stenzel, V. Schmidt, M. Roland, S. Diebels, F. Mücklich, 3d connectivity of eutectic si as a key property defining strength of al-si alloys, Computational Materials Science 120 (2016) 99-107.

[2] D. Bujoreanu, H. Dorez, W. Boutegrabet, D. Moussata, R. Sablong, D. Rousseau, Robust graph representation of images with underlying structural networks. application to the classification of vascular networks of mice's colon, Pattern Recognition Letters 87 (2017) 29-37.

[3] A. Aryanfar, S. Medlej, A. Tarhini, S. Damadi, A. Tehrani B., W. Goddard III, 3d percolation modeling for predicting the thermal conductivity of graphene-polymer composites, Computational Materials Science 197 (2021) 110650 .

[4] B. Prifling, D. Westhoff, D. Schmidt, H. Markoetter, I. Manke, V. Knoblauch, V. Schmidt, Parametric microstructure modeling of compressed cathode materials for li-ion batteries, Computational Materials Science 169 (2019) 109083.

[5] U. Bhardwaj, A. Sand, M. Warrier, Graph theory based approach to characterize self interstitial defect morphology, Computational Materials Science 195 (2021) 110474.

[6] B. Prifling, M. Ademmer, F. Single, O. Benevolenski, A. Hilger, M. Osenberg, I. Manke, V. Schmidt, Stochastic $3 \mathrm{~d}$ microstructure modeling of anodes in lithium-ion batteries with a particular focus on local heterogeneity, Computational Materials Science 192 (2021) 110354

[7] F. Dullien, Porous media: fluid transport and pore structure, Academic Press, 1979.

[8] P. Adler, J.-F. Thovert, Real porous media: Local geometry and macroscopic properties, Applied Mechanics Reviews 51 (9) (1998) 537-585.

[9] K. Michielsen, H. De Raedt, Morphological image analysis, Computer Physics Communications 132 (1-2) (2000) 94-103.

[10] É. Kaeshammer, L. Borne, F. Willot, P. Dokládal, S. Belon, Morphological characterization and elastic response of a granular material, Computational Materials Science 190 (2021) 110247.

[11] B. Prifling, M. Neumann, D. Hlushkou, C. Kübel, U. Tallarek, V. Schmidt, Generating digital twins of mesoporous silica by graph-based stochastic microstructure modeling, Computational Materials Science 187 (2021) 109934

[12] G. Hahn, M. Fleck, Automatic etch pit density analysis in multicrystalline silicon, Computational Materials Science 183 (2020) 109886.

[13] F. Thibault-Starzyk, I. Stan, S. Abelló, A. Bonilla, K. Thomas, C. Fernandez, J.-P. Gilson, J. Pérez-Ramírez, Quantification of enhanced acid site accessibility in hierarchical zeolites-the accessibility index, Journal of Catalysis 264 (1) (2009) 11-14.

[14] D. Do, L. Herrera, C. Fan, A. Wongkoblap, D. Nicholson, The role of accessibility in the characterization of porous solids and their adsorption properties, Adsorption 16 (1-2) (2010) 3-15.

[15] N. Nishiyama, T. Yokoyama, Permeability of porous media: role of the critical pore size, Journal of Geophysical Research: Solid Earth 122 (9) (2017) 6955-6971.

[16] Y. She, J. Lee, B. Diroll, T. Scharf, E. Shevchenko, D. Berman, Accessibility of the pores in highly porous alumina films synthesized via sequential infiltration synthesis, Nanotechnology 29 (49) (2018) 495703.

[17] V. Wernert, R. Bouchet, R. Denoyel, Influence of molecule size on its transport properties through a porous medium, Analytical Chemistry 82 (7) (2010) 2668-2679.

[18] M. Skaug, L. Wang, Y. Ding, D. Schwartz, Hindered nanoparticle diffusion and void accessibility in a threedimensional porous medium, ACS nano 9 (2) (2015) 2148-2156.

[19] J. Chaniot, M. Moreaud, T. Fournel, J.-M. Becker, The reachable volume fraction in porous media in the vicinity of percolation threshold: a numerical approach used on multi-scale boolean schemes, in: 2017 16th Workshop on Information Optics (WIO), IEEE, 2017, pp. 1-3.

[20] S. Broadbent, J. Hammersley, Percolation processes: I. crystals and mazes, in: Mathematical Proceedings of the Cambridge Philosophical Society, Vol. 53, Cambridge University Press, 1957, pp. 629-641.

[21] D. Stauffer, Introduction to percolation theory, Francis \& Taylor, 1985.

[22] M. Sahimi, Applications of percolation theory, CRC Press, 1994.

[23] E. Petersen, Diffusion in a pore of varying cross section, AIChE Journal 4 (3) (1958) 343-345.

[24] L. Holzer, D. Wiedenmann, B. Münch, L. Keller, M. Prestat, P. Gasser, I. Robertson, B. Grobéty, The influence of constrictivity on the effective transport properties of porous layers in electrolysis and fuel cells, Journal of Materials Science 48 (7) (2013) 2934-2952.

[25] D. Jeulin, M. Moreaud, Percolation of random cylinder aggregates, Image Analysis \& Stereology 26 (3) (2007) $121-127$.

[26] M. Neumann, C. Hirsch, J. Staněk, V. Beneš, V. Schmidt, Estimation of geodesic tortuosity and constrictivity in stationary random closed sets, Scandinavian Journal of Statistics (2019). 
[27] H. Vogel, Morphological determination of pore connectivity as a function of pore size using serial sections, European Journal of Soil Science 48 (3) (1997) 365-377.

[28] H.-J. Vogel, Topological characterization of porous media, in: Morphology of condensed matter, Springer, 2002, pp. 75-92.

[29] P. Dupuy, P. Austin, G. Delaney, M. Schwarz, Pore scale definition and computation from tomography data, Computer Physics Communications 182 (10) (2011) 2249-2258.

[30] J. Ohser, C. Ferrero, O. Wirjadi, A. Kuznetsova, J. Duell, A. Rack, Estimation of the probability of finite percolation in porous microstructures from tomographic images, International journal of materials research 103 (2) (2012) 184 191

[31] J. Serra, Image analysis and mathematical morphology, Academic Press, Inc., 1982.

[32] P. Soille, Morphological image analysis: principles and applications, Springer Science \& Business Media, 2013.

[33] J. Chaniot, Caractérisation morphologique efficace de matériaux par cartes de distance, Ph.D. thesis, University of Lyon (2019).

[34] J. Chaniot, M. Moreaud, L. Sorbier, T. Fournel, J.-M. Becker, Tortuosimetric operator for complex porous media characterization, Image Analysis \& Stereology 38 (1) (2019) 25-41.

[35] plug im!, plug im!: an open access and customizable software for signal and image processing (2018). URL https : //www . plugim. fr

[36] L. He, X. Ren, Q. Gao, X. Zhao, B. Yao, Y. Chao, The connected-component labeling problem: A review of state-of-the-art algorithms, Pattern Recognition 70 (2017) 25-43.

[37] J. Chaniot, M. Moreaud, L. Sorbier, D. Jeulin, J.-M. Becker, T. Fournel, Heterogeneity assessment based on average variations of morphological tortuosity for complex porous structures characterization, Image Analysis \& Stereology 39 (2) (2020) 111-128

[38] A. Baddeley, E. Jensen, Stereology for statisticians, Chapman and Hall/CRC, 2004

[39] G. Borgefors, Distance transformations in digital images, Computer Vision, Graphics, and Image Processing 34 (3) (1986) 344-371.

[40] P. Saha, G. Borgefors, G. S. di Baja, A survey on skeletonization algorithms and their applications, Pattern Recognition Letters 76 (2016) 3-12.

[41] F. Bini, A. Pica, A. Marinozzi, F. Marinozzi, A 3d model of the effect of tortuosity and constrictivity on the diffusion in mineralized collagen fibril, Scientific reports 9 (1) (2019) 2658.

[42] G. Matheron, Random sets and integral geometry, J. Wiley, New York (1975).

[43] J. Serra, The boolean model and random sets, in: Computer Graphics and image Processing, Vol. 12, Academic Press, Inc., 1980, pp. 99-126.

[44] S. Chiu, D. Stoyan, W. Kendall, J. Mecke, Stochastic geometry and its applications, John Wiley \& Sons, 2013.

[45] J. Kingman, Poisson processes, Wiley Online Library, 1993.

[46] A. Aubert, D. Jeulin, Estimation of the influence of second-and third-order moments on random sets reconstructions, Pattern Recognition 33 (6) (2000) 1083-1104.

[47] D. Jeulin, Morphology and effective properties of multi-scale random sets: A review, Comptes Rendus Mécanique 340 (4-5) (2012) 219-229, recent Advances in Micromechanics of Materials.

[48] M. Moreaud, J. Chaniot, T. Fournel, J. Becker, L. Sorbier, Multi-scale stochastic morphological models for 3d complex microstructures, in: 2018 17th Workshop on Information Optics (WIO), IEEE, 2018, pp. 1-3.

[49] M. Neumann, E. Charry, K. Zojer, V. Schmidt, On variability and interdependence of local porosity and local tortuosity in porous materials: a case study for sack paper, Methodology and computing in applied probability (2020) $1-15$

[50] K. Mecke, H. Wagner, Euler characteristic and related measures for random geometric sets, Journal of Statistical Physics 64 (3-4) (1991) 843-850.

[51] K. Mecke, Morphology of spatial patterns-porous media, spinodal decomposition and dissipative structures, Acta Physica Polonica. Series B 28 (8) (1997) 1747-1782.

[52] N. Roberts, M. Reed, G. Nesbitt, Estimation of the connectivity of a synthetic porous medium, Journal of Microscopy 187 (2) (1997) 110-118.

[53] H. Minkowski, Volumen und oberflache, volume 447, Math. Annalen 57 (1903).

[54] J. Jernot, P. Jouannot, Euler-poincaré characteristic of a randomly filled three-dimensional network, Journal of Microscopy 171 (3) (1993) 233-237.

[55] C. Arns, M. Knackstedt, W. Pinczewski, K. Mecke, Euler-poincaré characteristics of classes of disordered media, Physical Review E 63 (3) (2001) 031112

[56] J. Ohser, W. Nagel, K. Schladitz, The euler number of discretized sets—on the choice of adjacency in homogeneous lattices, in: Morphology of condensed matter, Springer, 2002, pp. 275-298.

[57] J. Ohser, W. Nagel, K. Schladitz, The euler number of discretised sets-surprising results in three dimensions, Image Analysis \& Stereology 22 (1) (2003) 11-19.

[58] M. Moreaud, B. Celse, F. Tihay, Analysis of the accessibility of macroporous alumino-silicate using 3d-tem images, 
in: Proceedings of Materials Science \& Technology 2008 Conference and Exhibition: MS\&T, Vol. 8, 2008, pp. 1153-64.

[59] A. Batista, W. Baaziz, A.-L. Taleb, J. Chaniot, M. Moreaud, C. Legens, A. Aguilar-Tapia, O. Proux, J.-L. Hazemann, F. Diehl, et al., Atomic scale insight into the formation, size, and location of platinum nanoparticles supported on $\gamma$-alumina, ACS Catalysis 10 (7) (2020) 4193-4204. 